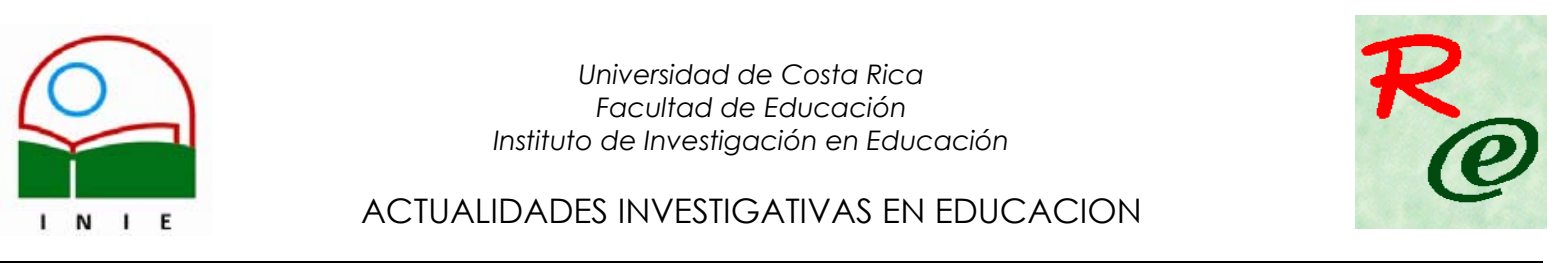

\title{
LA ACREDITACION COMO PROCESO DINAMIZADOR HACIA LA CALIDAD
}

\author{
ACCREDITATION AS A REVITALIZING PROCESS TOWARDS QUALITY
}

\author{
Belén Boville Luca de Tena ${ }^{1}$ \\ Nohemí Argüello Sosa ${ }^{2}$ \\ Noemí Guadalupe Reyes Castro ${ }^{3}$
}

\begin{abstract}
Resumen: Con metodología de estudio de caso se aborda la acreditación de un centro de Educación Superior mexicano, la Unidad Académica Multidisciplinaria de Comercio y Administración Victoria de la Universidad Autónoma de Tamaulipas. Mediante el estudio del proceso de evaluación se trata de descubrir cómo ha generado el dinamismo que permite una mejora sustancial de la calidad. Los hallazgos ponen de manifiesto un modelo de gestión que privilegia liderazgo compartido, planeación estratégica, autoevaluación y trabajo en equipo en torno a una visión común. Se identifica una planta docente profesionalizada y programas de estudio con enfoque integral, complementado con actividades de investigación, cultura y deporte. Comprometidos con un proceso de mejora contínua, la consolidación de metas a corto y largo plazo está íntimamente relacionada con el sentido de pertenencia de sus miembros a esa organización. La corresponsabilidad ante el éxito o el fracaso es una característica clave en el éxito de esta comunidad educativa.
\end{abstract}

\section{Palabras clave: EVALUACION/ AUTOEVALUACIÓN/ CALIDAD/}

Abstract: This educational survey is about the process of certification and assessment practised by a Mexican University Faculty of Administration and Commerce. As a case study, the authors have worked in the "Unidad Académica Multidisciplinaria de Comercio y Administración de Victoria"-UAMCAV-, of the Universidad Autónoma de Tamaulipas, in the Northeast of Mexico. The research identifies a model of management that favours shared leadership, strategic planification, autoevaluation and team work around a a common vision. A professional staff, programs of study and integrated approach complemented with activities of investigation, culture and sport allows the excellence certified by the Evaluation Authorities. Continuous improvement and the consolidation of goals in the short and long term is related to the sense of belonging to this organization. Corresponsability to either success or failure is a key characteristic in the success of this educational community.

Key words: EVALUATION/ AUTOEVALUATION/ QUALITY/

\footnotetext{
${ }^{1}$ Doctora en Geografía e Historia por la Universidad Complutense de Madrid. Master en Educación Ambiental (UNED/UNESCO) Escritora y Periodista. Profesora de Tiempo Completo en la Universidad Autónoma de Tamaulipas (México). Miembro del Cuerpo Académico de Historia y Sociología de la Educación.Correo electrónico: belenbovillelt@yahoo.com

2 Candidata a Doctor en Educación por la Universidad Autónoma de Tamaulipas. Maestría en Educación. Licenciada en Biología. Profesora de Tiempo completo en bachillerato (educación media superior) y de tiempo parcial en secundaria (educación media básica). Correo electrónico: nohemiarguello@yahoo.com.mx

${ }^{3}$ Licenciada en Educación Básica con Maestría en Formación Docente. Es Coordinadora General Estatal del Programa Escuelas de Calidad. y candidata a Doctora en Educación por la U.A. de Tamaulipas. Correo electrónico: noemireyesc prim@hotmail.com
}

Artículo recibido: 10 de febrero, 2006

Aprobado: 27 de marzo, 2006 


\section{Introducción.}

Las razones que han conducido a la realización de esta investigación se originan en la pertinencia educativa: la adecuación de la Universidad a una sociedad cambiante y dinámica, que busca, además de la diversidad en la oferta educativa, la garantía de una educación de calidad. Aunque las condiciones y la certificación de la calidad sean discutibles, lo que generó en su día un debate que aún está abierto, son suficientemente alentadoras como para dinamizar procesos de reforma y renovación y para estimular la participación voluntaria de los actores involucrados en ella.

Como la Universidad pública en México ha sufrido una larga travesía del desierto, de la que está saliendo paulatinamente como veremos a continuación, está en el ánimo de los investigadores reivindicar la educación pública en México y tomar consecuentemente y como ejemplo, un caso exitoso donde la oferta de educación superior es de calidad y permite esa adecuación en la relación entre educación y sociedad.

Hoy en día las imposiciones de la globalización no son ajenas al mundo universitario. Si ha habido una rápida adaptación del mundo empresarial y productivo a las exigencias del mercado, es necesario que la Universidad se adapte a todo esto como sociedad del conocimiento (Allende, 2001). Ello demanda una adecuación y la pertinencia educativa: que la Universidad corresponda a estos cambios sociales, y concretamente la Universidad pública. Este imperativo, adoptado por Europa en el Plan de Boloña (Bricall, 2000) se ha ido incorporando a las Universidades mexicanas mediante la reforma universitaria, tratando de responder a la nueva situación e intentando salir del marasmo al que se había abocado a la Educación Superior en México en el último tercio del pasado siglo (Levy, 1995).

En las últimas décadas la universidad mexicana ha sido sometida a presiones demográficas y políticas intensas. Clientelismo, demagogia y un sentido de servicio social y público al margen de los procesos de calidad supuso convertirla en pocos años en una universidad de masas, adaptándose defensivamente, hinchando sus estructuras administrativas y académicas con criterios escasamente pedagógicos y sí que dictados por las necesidades políticas del momento. Surgió así un sistema grande y diversificado, de calidad muy desigual (Kent, 2002), y que expandió formidablemente la corrupción. Este tipo de sistema universitario no respondía a los imperativos modernizadores de una sociedad en desarrollo y ha sido definitivamente un freno en una época supuestamente optimista.

Como consecuencia de ello, se presentó una proliferación de escuelas privadas de baja calidad, que respondían con mediocridad a las necesidades sociales en materia de formación. La escasa calidad fue además provocada por la relajación de las políticas 
gubernamentales de acreditación y control de nuevas instituciones. Estos centros se han preocupado fundamentalmente por atender una demanda de certificados superiores (Levy, 1995).

Hoy en día México presenta un problema estructural de desajuste entre la oferta potencial de egresados del sistema educativo y la demanda efectiva de trabajadores y cuadros técnicos (Urquidi, 1996). Este problema no se limita a las áreas de planeación sino que también corresponde con un cambio de mentalidad y el paso de macroestructuras educativas a una Universidad más flexible (Navarro, 2000) y que tenga en cuenta las necesidades sociales, con programas accesibles de educación contínua y la vinculación necesaria entre el mundo educativo y profesionista/empresarial.

En las negociaciones de la Organización Mundial de Comercio (OMC) de noviembre de 2001 se planteó el objetivo de liberalizar los servicios públicos, incluidos los servicios educativos (OMC, 2001). En el capítulo V del Informe 2001 del Director General de la Organización Mundial para el Comercio se abordan las cuestiones y críticas a una supuesta liberalización de los servicios de educación y salud (AGCS o Acuerdo General sobre el Comercio de Servicios). Aunque se deja completa discrecionalidad a los gobiernos para legislar y desarrollar políticas soberanas en estos aspectos, también se deja la puerta abierta para una liberalización de este tipo de servicios que pasarían a formar parte del mercado mundial, como cualquier otra mercancía. En este sentido, la competitividad y capacidad de iniciativa mostrada por algunas universidades privadas en el ámbito de la globalización (Tyack, 2000) confirma los temores sobre las repercusiones negativas que ello pueda tener sobre las instituciones de Educación Superior en el ámbito público. Es así necesario crear una cultura de la educación pública que permita afrontar los retos de la globalización y sus consecuencias liberalizadoras que llevarían a considerar ésta como un servicio más. Y esta cultura de la educación pública solo se puede generar partiendo de la excelencia educativa y de los programas de calidad. Razón por la cual se presenta el presente trabajo sobre la Unidad Académica de Comercio en la Universidad Autónoma de Tamaulipas (México), que se inscribe dentro del proceso generalizado de búsqueda de la calidad y acreditación que se comenzó en México a principios de los 90.

La larga travesía del desierto en México, significó, en los 90, una apuesta por la calidad y la reforma. Esta implica un verdadero ejercicio de autocrítica y la necesidad de iniciar procesos de evaluación, que no solo han venido a certificar situaciones concretas, sino que han contribuido incluso a la "mutación" del sistema de educación superior. La acreditación no ha sido entonces la culminación de un proceso, sino el comienzo del mismo, por su papel 
como instrumento de gestión y porque tanto evaluación como acreditación han sido vectores de cambio (Didou, 2005). En los años 90, la firma del Tratado de Libre Comercio de América del Norte (TLCAN), sirvió como pretexto para estimular procesos de calidad, pero pronto éstos se significaron y singularizaron al margen de la situación internacional. Comenzaron entonces a instalarse dispositivos para detectar la calidad de la educación superior. El mecanismo de vinculación con programas adicionales de financiamiento fue el motor inicial para provocar esta mejora. En paralelo, el gobierno fomentó la creación de asociaciones civiles encargadas de acreditar la calidad de los programas de estudio y los procesos de gestión, así como de certificar a los actores, tanto académicos, como estudiantes y egresados. Así, en un sistema de Educación Superior que en general se podía calificar como deficiente (salvo honrosas excepciones) la evaluación y acreditación fueron auténticas soluciones técnicas, diseñadas para incidir en la organización de las instituciones. Su legitimidad fue entonces funcional y pasó al contexto nacional como forma de conducción, gestión que tanto en su conformación como en sus instrumentos, expresaba una voluntad de modernización. Así, tanto la evaluación como la acreditación impulsaron el reordenamiento del sistema superior y sirvieron como mecanismo de cambio que obviaba una reflexión en profundidad. Tal como establece Sylvie Didou,

Los dispositivos complementarios de evaluación y de acreditación, aunados progresivamente unos a otros, constituyen en México uno de los sistemas más complejos y diferenciados de aseguramiento de calidad en América Latina: abarca actualmente individuos, programas, procesos de investigación, de docencia e incluso de administración. Lo animan agencias gubernamentales, asociaciones civiles, agencias y organismos internacionales, especializados en la medición, vía la supervisión de calidad institucional o disciplinaria y la certificación de las competencias o de los conocimientos individuales (Didou, 2005, p.32)

Estamos ante una preocupación que se extiende al mundo docente y académico. Puntos de vista y análisis recientes de investigaciones y autores interesados en la materia, dan a conocer los procesos que han desarrollado las universidades para adaptarse a los cambios y necesidades de la demanda, sin perder su identidad fundamental que está basada, fundamentalmente, en el carácter público del servicio; además de "...desarrollar una capacidad de adaptación que se muestra en sus características de organización". (Clark, 1983, p. 264) 
Como resultado de estos procesos, tratando de lograr una mejor oferta educativa se piensa en la dirección e intensidad del proceso de planeación, evaluación y desde luego en la calidad de la educación superior. Con un sentido de la responsabilidad y de las necesidades que demanda esta sociedad cambiante, la Universidad Autónoma de Tamaulipas, objeto de nuestro estudio, creó el "Plan estratégico 2003-2007 Millenium III", que es el marco de referencia que da sentido a la renovación en función de la complejidad de las dinámicas sociales, económicas, políticas y académicas del contexto situacional en el tercer milenio. Se valora así la planeación participativa como un elemento fundamental para que la comunidad universitaria en sus diferentes ámbitos y según sus propias necesidades presentes y futuras, bosqueje el camino a seguir para consolidar las metas de calidad educativa, algo que sólo se puede realizar mediante una implicación directa y una corresponsabilidad. Así es ilustrativo que el documento rector señale, entre otros atributos que ha de conseguir la universidad... "la calidad creciente en sus procesos y productos, que le permita acceder a la acreditación y certificación nacional e internacional" (Lavín, 2003, p.4).

La reforma universitaria que significa Millenium III se fundamenta en el Programa Nacional de Educación 2001-2006, en donde se señalan líneas claras y estrategias precisas para el sistema educativo: el camino seguro a una oferta educativa con pertinencia, equidad y calidad... "incluyente e integralmente formativa, que constituya el eje fundamental del desarrollo cultural, científico, económico, tecnológico y social de México" (SEP, 2001, p. 71). Cabe señalar otro de los atributos que con el diseño de estrategias adecuadas, caracterizará a la institución estudiada... “Una estructura de organización, gestión y toma de decisiones, con base en un marco normativo actualizado, con amplia participación de la comunidad a través de órganos colegiados" (Lavín, 2003, p. 4).

El caso particular de la Universidad Autónoma de Tamaulipas, donde se promueve un cambio organizacional que eleve el estatus de los resultados, es motivo de cuestionamientos y análisis acerca del nivel de logro y los cambios experimentados a partir de la reforma mencionada. Esta investigación vuelve la mirada hacia la Unidad Académica Multidisciplinaria de Comercio y Administración Victoria (UAMCAV de ahora en adelante); la cual, en el contexto universitario, goza de aceptación y se considera una comunidad educativa que, inmersa en un proceso de cambio, trata de adecuar su estructura a las necesidades de la demanda y del entorno, en congruencia con la misión de la comunidad universitaria a la que pertenece. 
Esta unidad académica ha generado un programa de mejoramiento continuo en los ámbitos normativo, administrativo y académico que le ha significado reorientar la dinámica institucional con la intención de fortalecer el proceso de enseñanza. Lo anterior se concreta en consolidar un trabajo colegiado en academias, repensar los enfoques psicopedagógicos de los programas de estudio, transformar los procesos de evaluación y acreditación del aprendizaje, así como el establecimiento de un nuevo esquema de relación interpersonal entre la dirección, docentes, trabajadores y alumnos, con base en una cultura sustentada en la filosofía de calidad. La reforma curricular se realiza en todas las unidades académicas de la universidad; sin embargo, en este centro educativo se realiza, adicionalmente, un proceso de mejora institucional. Una dirección eficaz, un grupo académico de docentes comprometido y los alumnos con alta disponibilidad al aprendizaje, hacen la diferencia; es decir, construyen día a día una identidad institucional que distingue su labor de excelencia.

El interés por encontrar información relevante acerca de esta unidad académica se debe a su éxito en los procesos de autoevaluación, pues han sido éstos los que han servido para conseguir la Certificación y Acreditación de dos carreras de Licenciatura de las tres que se imparten en la UAMCAV: la de Administración y la de Contador Público. Este ha sido el motivo principal para seleccionar el objeto de estudio, y como decíamos anteriormente, crear una cultura de la educación pública. El que sea una institución exitosa nos ha llevado a diseñar una investigación participativa que tenga como resultado la identificación de elementos, factor de transformación en esa comunidad educativa.

Aunque se ha razonado la necesidad de una educación de calidad y la disposición institucional que promueva la misma, tanto desde la Secretaría de Educación Pública, como desde los organismos rectores de las Universidades, los procesos de calidad, para que sean genuinos y auténticos, requieren de una internalización, es decir de la adopción sincera y dispuesta de todo el personal involucrado, tanto directivos y planeadores como académicos, personal administrativo y estudiantes. En este caso concreto, la decisión de la Autoevaluación -como tal ejercicio autocrítico- se adopta voluntariamente por parte del equipo directivo- que la traslada al conjunto de la Unidad académica y consigue que tanto profesores como alumnos y asistentes se involucren en el proceso de una manera entusiasta. Este ha sido el mecanismo que ha dado luego como resultado la Acreditación, pero viéndolo desde el otro lado, es la misma Acreditación -la promesa de una Certificación de Calidad a nivel nacional- la que motiva a todo el personal involucrado. De ahí que Acreditación y Autoevaluación se retroalimenten. 
El presente informe está relacionado con el análisis de este proceso de acreditación, desarrollo y primeros resultados y la intervención de organismos evaluadores: los Comités Interinstitucionales para la Evaluación de la Educación Superior (en adelante CIEES) y el Consejo de Acreditación en la Enseñanza de la Contaduría y Administración, A.C. (en adelante CACECA), cuya participación es de gran relevancia para el logro de propósitos. El proceso de autoevaluación se inició a principios de 2002, siguiéndose las premisas marcadas por CIEES y por CACECA, obteniéndose en el 2004 la acreditación de las dos carreras de Licenciatura mencionadas.

En el proceso de mejora adoptado por la UAMCAV, la autoevaluación constituye la base firme para diseñar estrategias y acciones. $Y$ así se ha estimulado desde dentro tanto los sistemas de autoevaluación como la evaluación interinstitucional. Así como para muchas instituciones las evaluaciones $\mathrm{e}$ incluso las autoevaluaciones crean resquemor $\mathrm{y}$ desconfianza (Glazman, 2001), para esta institución, sin embargo, la evaluación externa no es un obstáculo para sus acciones y el logro de sus metas.

La evaluación externa no es antitética de la autonomía y el desarrollo académico, sino que más bien conforma un todo a partir de la transparencia, la productividad, la responsabilidad y el juicio de pares. De este modo, la calidad, la eficiencia y la pertinencia, pasan a ser los nuevos patrones de juicio de la acción universitaria. (Naishtat 2001, p.20)

Este análisis técnico pretende localizar fortalezas, logros y debilidades en la construcción y desarrollo del proceso de acreditación. Es una experiencia vivida por la comunidad educativa y por lo cual vale la pena identificar circunstancias y factores en interacción que les condujeron a las metas propuestas. Este artículo parte de la investigación "Evaluación y Acreditación en la U.A.T.: la UAMCAV como modelo de excelencia educativa" que se realizó en Tamaulipas (México) en 2005 (Boville, 2005).

\section{Metodología}

Nos sirve para esta investigación la metodología del estudio de caso (Mc Kernan, 1999) entendido como la consecución de resultados y datos que se presentan interpretados como un caso único, incluyéndose la información conseguida con el trabajo de campo redactado al fin de un ciclo de acción.

El estudio de caso ha significado la revisión -además de la literatura especializada en temas de evaluación y de investigación educativa- de los documentos rectores respecto a la 
reforma en la universidad estudiada, los documentos de gobierno interno de la unidad académicas y todos aquellos que han formado parte del proceso de autoevaluación y evaluación (documentos de CIEES y CACECA).

En cuanto al estudio de caso, se han diseñado diferentes instrumentos de tipo cualitativo, cimentados en las técnicas de la observación participante, la entrevista en profundidad, los cuestionarios y el grupo de discusión. La riqueza obtenida en diferentes grupos de trabajo con distintos cometidos ha permitido el intercambio de información y el avance paulatino de la investigación contrastándose los diferentes aspectos de la misma.

Respecto a los mecanismos de control de la evaluación de las instituciones citadas se elaboró una muestra en la que se consideraron 24 docentes. Todos estos profesores eran responsables de diferentes programas o áreas, y durante el proceso de acreditación han tenido por encargo, en colaboración con el personal, atender a las recomendaciones de CACECA y superar los retos y las recomendaciones. Su intervención se ha considerado clave para guiar el proceso hacia la consecución de los fines.

El enfoque cualitativo adoptado dio como resultado información relacionada con una situación específica en un contexto plenamente identificado: la comunidad educativa de la UAMCAV. Esta información permite describir, con base en lo aportado por los protagonistas, la situación en el momento de la investigación, lo que permite “...interpretar, inferir y evaluar, si así se desea." (Schmelkes, 2002, p. 34).

En el análisis de los resultados ha sido fundamental la agrupación y procesamiento de datos mediante la triangulación de información, que sirvió para la obtención de relatos, interpretaciones y conclusiones sobre los hechos concretos. Esta fase fue determinante para la organización creativa del conjunto del material, que dio como resultado una narración interpretativa y descriptiva del proceso de cambio institucional.

\subsection{Instrumentos}

Se diseñaron 24 formatos para entrevistas semiestructuradas, cuyo contenido (interrogantes) está relacionado con las 8 variables y categorías correspondientes, en las que se inscriben las recomendaciones de CACECA como resultado de la evaluación.

El propósito de los instrumentos es identificar fortalezas y debilidades en el proceso de acreditación. Desde la perspectiva de los informantes, de sus interpretaciones que ponen de manifiesto el modo en que ellos... "ven y conciben los procesos de innovación educativa". (Mckernan, 1996, p.13). 


\subsection{Variables}

Se tomaron en cuenta todas las variables y categorías que guiaron el proceso de acreditación y que fueron definidas por CACECA en la implementación del proceso.

\begin{tabular}{|c|c|}
\hline VARIABLES & CATEGORÍAS \\
\hline 1. PROFESORES & $\begin{array}{l}\text { 1.1 } \text { Formación } \\
\text { 1.2 Experiencia } \\
\text { 1.3 Selección } \\
\text { 1.4 Calificación } \\
\text { 1.5 Asignación de carga }\end{array}$ \\
\hline 2. ESTUDIANTES & $\begin{array}{l}\text { 2.1 Admisión } \\
\text { 2.2 Evaluación de la calidad en el desempeño } \\
\text { 2.3 Apoyo académico } \\
\text { 2.4 Reglamento de titulación } \\
\text { 2.5 Otros: becas, estímulos, vinculación familia- } \\
\text { escuela. }\end{array}$ \\
\hline $\begin{array}{l}\text { 3. PROGRAMA DE LICENCIATURA } \\
\text { ADMINISTRACIÓN, CONTADOR } \\
\text { PÚBLICO, INFORMATICA. }\end{array}$ & $\begin{array}{l}\text { 3.1 } \text { Currículum - Generales } \\
\text { 3.2 Entorno - relaciones } \\
\text { 3.3 Plan de estudios } \\
\text { 3.4 Objetivo cognitivo o conocimiento fundamental } \\
\text { 3.5 Otros. Titulación-deserción, reprobación, } \\
\text { servicio social. }\end{array}$ \\
\hline 4. FORMACIÓN INTEGRAL & $\begin{array}{l}\text { 4.1 Actividades culturales } \\
\text { 4.2 Deportivas } \\
\text { 4.3 Congresos } \\
\text { 4.4 Desarrollo empresarial } \\
\text { 4.5 Académico científico }\end{array}$ \\
\hline 5. RECURSOS FINANCIEROS & $\begin{array}{l}\text { 5.1 Aportaciones-donativos } \\
5.2 \text { Ingresos de proyectos especiales } \\
5.3 \text { Eficiencia } \\
\text { ○ Proceso de la administración } \\
\text { ○ Financiamiento } \\
\text { ○ Otros. Apoyo sector productivo, vínculos, } \\
\text { utilización de instalaciones. }\end{array}$ \\
\hline
\end{tabular}




\begin{tabular}{|l|l|}
\hline 6. RECURSOS-EFICIENCIA & $\begin{array}{l}6.1 \text { Infraestructura } \\
6.2 \text { Procesos y equipo } \\
6.3 \begin{array}{l}\text { Otros. Biblioteca, recursos de información, } \\
\text { estudiantes por grupo de titulación. }\end{array}\end{array}$ \\
\hline 7. EXTENSIÓN INVESTIGACIÓN & $\begin{array}{l}\text { 7.1. Proyectos de extensión, proyectos de } \\
\text { investigación, investigaciones publicadas. }\end{array}$ \\
\hline 8. INFORMACIÓN ADICIONAL & $\begin{array}{l}8.1 \text { Personal administrativo, de servicios y apoyo. } \\
8.2 \text { Otros. Evaluaciones periódicas. }\end{array}$ \\
\hline
\end{tabular}

\subsection{Desarrollo}

La documentación aportada ha permitido comprobar que los dos organismos evaluadores, CIEES y CACECA, realizan periódicas evaluaciones, cuyo resultado ha sido una retroalimentación y un constante diálogo que ha permitido redefinir las metas, establecer los procedimientos más adecuados y acertar en las acciones.

El análisis permitió identificar las líneas de acción a considerar a fin de cumplir con las metas organizacionales programadas, los responsables de los programas y áreas y las observaciones y recomendaciones realizadas.

Posteriormente, se diseñaron los instrumentos para recabar la información necesaria. Las entrevistas se efectuaron a cada uno de los encargados de orientar el cumplimiento de las acciones para atender y solventar las recomendaciones hechas por CACECA, y relacionadas con los programas de Contaduría Pública y Licenciatura en Administración.

Al concluir la recolección de datos se llevó a cabo la sistematización de la información, producto del análisis de cada una de las entrevistas.

\section{Análisis de Resultados.}

El estudio y análisis del diagnóstico de CIEES y del proceso de acreditación de CACECA en la UAMCAV, identificó algunas fortalezas y áreas de oportunidad de la comunidad educativa, así como factores que incidieron de manera positiva o negativa en los proyectos instrumentados para la mejora continua de esta Unidad Académica. De este análisis se identificaron algunas actividades y factores relevantes que pueden coadyuvar el proceso de acreditación de otros centros o instituciones educativas.

La información que aquí se presenta contiene datos relacionados con las recomendaciones de CACECA, la participación de la comunidad educativa en el proceso de Volumen 6, Número 1, Año 2006, ISSN 1409-4703 10 
acreditación, las sugerencias y apreciaciones que tienen a bien hacer los entrevistados. Las observaciones relevantes están organizadas en ocho variables y sus categorías respectivas, considerando lo que establece CACECA para el proceso de acreditación.

\subsection{Profesores}

La formación y contratación son las categorías que reciben la más alta puntuación por parte del organismo evaluador. Se analiza la procedencia académica y la forma en la que se contrató al personal docente, que se realiza mediante convocatorias publicadas y un comité de selección. Uno de los aspectos que consideraban mejorable es la investigación, por lo que se invita a participar en proyectos de investigación relacionados con programas académicos curriculares como una manera de integración y realimentación de los mismos. La problemática considerada para las líneas de investigación está relacionada con los planes de estudios, ésta se somete a debate y consenso de la academia.

Se contempla la formación continua del profesorado y su necesidad de promoción. Existen 32 catedráticos con tiempo completo participando activamente en el programa de investigación. Para ello se aportan recomendaciones de apoyo institucional en base a becas del Programa de Mejoramiento del Profesorado de Educación Superior, dedicado a la formación continua de este personal, y convenios interinstitucionales para la formación docente. El organismo evaluador también considera el establecimiento de redes de colaboración para la realización de investigaciones y la publicación de obras. Dentro de la formación del profesorado se incluye un programa docente con cursos pedagógicos y disciplinarios que introduzca en las aulas los procesos de enseñanza/aprendizaje y que familiarice a los docentes con los elementos básicos del constructivismo. Sin embargo y, al margen de los imperativos pedagógicos, los cursos del programa de formación docente se diseñan de acuerdo a las necesidades de los docentes, expresadas en las encuestas y en las reuniones de academia, elementales para conocer el funcionamiento.

En cuanto a la evaluación de los docentes, ésta se realiza mediante cuestionario contestado por los alumnos. Entre las distintas averiguaciones sobre su proceder como docentes, también se considera su trabajo en otros programas como investigación, proyectos de extensión, lo que da a dicha evaluación un carácter más integral que contribuye a la generación de una comunidad educativa y al sentimiento de pertenencia a la misma. 


\subsection{Estudiantes}

En cuanto a la admisión, el programa de inducción para estudiantes de nuevo ingreso es obligatorio. Las primeras becas asignadas son en función de los estudios socioeconómicos de los alumnos y son concedidas por controlaría.

La mayoría de los alumnos tienen una mentalidad no emprendedora, la cual representa un factor a vencer para lograr formarlos según las características descritas en el perfil del egresado en la UAMCAV. Este cambio de mentalidad va a ser una constante a lo largo de todo el programa de estudios y las actividades complementarias. Así, el plan de estudios por licenciatura considera un programa de actividades integrales que incluye desarrollo de habilidades comunicativas en talleres (oratoria, teatro...) y organización de eventos, como el festival de lectura. Los alumnos manifiestan un compromiso de trabajo y valoran la profesionalización docente, y así aprecian bastante el hecho de que algunos profesores no sean del ámbito académico y sí del profesional, de donde creen pueden obtener más información acerca del trabajo y las empresas.

En cuanto a la orientación hacia la investigación, el valor de los trabajos de investigación se aumentó en las diferentes asignaturas a 30\%, 40\% o 50\%. Anteriormente se daba más importancia al progreso en función del conocimiento de las materias.

Se ha optado por la concentración de los diferentes eventos extracurriculares (simposio, conferencias, etc.) de los diferentes programas académicos en un evento magno para hacer más práctica su realización y al mismo tiempo cubrir las metas que marca el organismo acreditador. Ejemplo de lo anterior es el Congreso CONFIA (Contaduría, Finanzas, Informática y Administración), organizado por los estudiantes de manera exitosa y realizado en marzo del 2005.

Los alumnos consiguen apoyo académico mediante el programa de asesoría académica y remedial, donde se vigila, se da seguimiento, se capacita y se documentan los resultados. Estos programas cuentan con la participación de becarios en apoyo a los alumnos en asesoría.

En cuanto a la titulación, ha habido un aumento del porcentaje de egresados titulados al permitir a todos los alumnos presentar su examen profesional una vez aprobado el examen del CENEVAL, que es el organismo evaluador de la educación media superior y superior.

Respecto a la relación familia-escuela se muestra un seguimiento constante de los alumnos, y una relación directa con los padres de familia. Al inicio del ciclo escolar se 
organiza una reunión especial para padres con fines informativos, envío de calificaciones a domicilio, cita a padres en caso necesario y entrevistas con padres a solicitud de ellos.

\subsection{Programas de Licenciatura: Administración, Contador Público e Informática.}

Las necesidades curriculares tales como la formación docente se detectan por medio de encuestas y opiniones expresadas en reuniones de academia. De ahí surgen las iniciativas para implementar cursos y talleres. Estas reuniones de academia están instituidas con sistema de registro y control (hay una participación de más del $40 \%$ de los profesores y una regularidad en sus reuniones y acuerdos).

Hay movilidad y comunicación con otras instituciones de educación superior lo que permite apoyar la formación docente mediante intercambios y estancias. La relación con diferentes áreas de gobierno y organismos no gubernamentales es amplia y constante y con ello se consigue ofrecer a los alumnos oportunidades de relación con el sector productivo. Las empresas han apoyado con software relacionado con el programa académico.

Respecto a los planes de estudio, los criterios para revalidaciones, equivalencias, acreditación y reconocimientos incluyen programas cursados en otras instituciones con objetivos, bibliografía, etc. También se presenta el perfil del egresado y los objetivos educativos. Se ha solicitado un aumento del nivel de inglés, la meta es el TOEFL $=350$ apoyada por clases extras.

En cuanto al Objetivo cognitivo los conocimientos fundamentales requeridos por CACECA están incluidos en los planes de estudios de las licenciaturas en Administración y Contador Público. El programa de estudios ha incluido asignaturas relacionadas con la investigación.

La oferta de posgrados en la unidad motiva a los egresados de licenciatura a titularse.

La base de datos permite el análisis de los porcentajes ingreso-egreso-titulación y el seguimiento de egresados. El porcentaje de reprobación ha disminuido considerablemente después del inicio del proceso de acreditación.

\subsection{Formación Integral}

En cuanto a Servicio Social, actividades culturales, deportivas, académicas y programas de desarrollo empresarial, los proyectos de desarrollo empresarial se han implementado con el software necesario para sistematizar la información resultante del 
mismo. El programa de desarrollo empresarial "Emprendedores" se ha impreso y organizado en carpetas rotuladas con las diferentes etapas del programa. La persona encargada de este tipo de programas es creativa, con capacidad de empatía con los alumnos y organizada.

Los programas que requieren la participación directa de los alumnos como "Emprendedores" necesitan de un seguimiento muy cercano, a fin de asesorar y animar a los alumnos a participar en ellos. La participación de los alumnos en programas que fomenten la creatividad es obligatoria, al menos en una primera etapa, para alcanzar las metas de CACECA.

Se incluye educación física en los programas de estudio y así se fomenta la participación en actividades deportivas. El viernes de cada semana se destina a tutorías, deporte, cultura. El control es por formatos de inscripción, lista de asistencia y base de datos para seguimiento.

\section{5. Recursos Financieros}

En los procesos de la administración, el Director se encarga de entablar relaciones y establecer convenios con instituciones de gobierno y particulares que representen fuentes de financiamiento. Este financiamiento se realiza mediante el trabajo conjunto del Contralor, el Director y el titular de la coordinación de planeación, que definen los proyectos necesarios y factibles congruentes a largo plazo con el trabajo de la unidad académica. Mediante este procedimiento se consigue que determinadas actividades, interesantes por su carácter investigativo o por ser parte del programa docente, se integren al programa de la unidad académica, garantizándose su desarrollo gracias precisamente a su rubro económico, lo que da recursos suplementarios a la unidad y la conecta de nuevo con el mundo productivo.

\section{6. Recursos-Eficiencia}

En la dotación de infraestructuras, el presupuesto de egresos contempla la construcción de rampas, aulas para clases faltantes y una cafetería adecuada para prestar buen servicio a los alumnos. En cuanto al equipo de personal, la participación del personal administrativo y de apoyo es del 100\%, alcanzado mediante reuniones de información y concienciación.

Se realizan entrevistas con el personal responsable de las áreas para confirmar los cambios y mejoras en el desempeño y se le da valor cualitativo al rendimiento del personal.

Otras cuestiones relacionadas con la biblioteca y los recursos de información nos permiten ver la colaboración de todos. El acervo bibliográfico de los diferentes programas 
académicos se enriquece con los libros donados por los egresados, requisito para la titulación. El acervo bibliográfico en la biblioteca se mantiene actualizado mediante un sistema con procedimientos que facilitan una revisión permanente. El servicio de estantería abierta, un procedimiento moderno, empezó a funcionar a finales del ciclo escolar 20042005, lo que brinda autonomía a los usuarios. El acervo bibliográfico responde a los programas de estudio, y se incrementa mediante las solicitudes, que se presentan en formatos diseñados ex profeso.

\subsection{Extensión Investigación}

El Plan de desarrollo institucional incluye líneas y proyectos de investigación cuyo desarrollo está sujeto a una normativa. Los proyectos de extensión están orientados por propósitos específicos congruentes con la metodología de evaluación de los mismos. La información generada permite la retroalimentación de los proyectos y evaluar su pertinencia y adecuación con las demandas sociales. En el Programa de Extensión, a los diferentes proyectos se les asigna un responsable que coordina las actividades del mismo. Se organizan equipos de estudiantes para atender casos específicos, relacionados con las asignaturas de los programas de estudio. La participación es de un $80 \%$ a un $90 \%$.

\subsection{Información Adicional}

En cuanto al Personal administrativo, de servicios y de apoyo, los programas para la formación o actualización de este personal se diseñan de acuerdo a necesidades específicas del personal. Los expedientes del personal de esta área permiten una visión clara de su profesionalización y desempeño. Existe un ambiente de confianza y flexibilidad en el desempeño de su trabajo.

Respecto a otras cuestiones como evaluaciones a alumnos, egresados, profesores, currículum y administración, en el programa de "Entorno e Impacto Social de los Programas Educativos" se aplican encuestas a empleadores y a egresados. La encuesta a empleadores incluye los datos generales del empleador y el centro de trabajo: régimen, área productiva, tamaño, así como tres preguntas cerradas con cinco opciones y tres preguntas abiertas. Las preguntas cerradas están relacionadas con la opinión del empleador, sobre el desempeño de los egresados de la Unidad Académica. Se busca la descripción de diferentes atributos personales: iniciativa, creatividad, presentación personal, puntualidad, actitud positiva, respeto, sociabilidad, compromiso con la empresa, responsabilidad; y profesionales: conocimientos básicos, especializados, de otra lengua, de paquetes Volumen 6, Número 1, Año 2006, ISSN 1409-4703 15 
computacionales, razonamiento lógico y analítico, habilidad para aplicar conocimientos, para tomar decisiones, para encontrar soluciones, para buscar y procesar información, para trabajar en equipo, para dirigir/coordinar, disposición para aprender, para el manejo de riesgos; habilidad para relacionarse con los demás, para la comunicación oral y escrita y la identificación con la empresa/institución. Las preguntas abiertas están relacionadas con la opinión de los empleadores sobre las desventajas que observan en los egresados de la UAMCAV, con recomendaciones para mejorar la formación de los egresados tanto en áreas de conocimientos como en el desarrollo de habilidades.

\section{Sugerencias para lograr mayor eficiencia en procesos que conducen a la acreditación}

A lo largo de la investigación, tanto en cuanto al contacto directo con responsables académicos y administrativos, como con los otros participantes del equipo investigador se han podido contrastar los diferentes aspectos que implica un proceso de acreditación y también cómo, tanto el organismo CIEES como CACECA, están a la altura de lo que es un proceso de evaluación entendido como una constante renovación y permanente actualización, muy lejos de los errores que se cometieron en el pasado cuando se inicia la cultura de la evaluación (Glazman, 2001).

Ya que nuestra investigación ha sido contrastada y triangulada por los distintos equipos de trabajo, estamos en la condición de hacer una serie de propuestas de tipo práctico para todos aquellos que se embarquen en la aventura de la acreditación.

Son las siguientes:

- Resolver todas las dudas que surjan durante el curso que ofrecen los organismos acreditadores.

- Solicitar a CIEES que imparta un curso-taller para involucrar, informar y concienciar de manera más completa a la comunidad educativa sobre el proceso.

- Considerar que el nivel de disposición para participar en el proceso de acreditación, es directamente proporcional al grado de información e involucramiento que se tiene sobre el mismo.

- El ambiente generado por el proceso de acreditación favorece en el personal el sentido de pertenencia a la institución lo que se refleja en su actitud positiva.

- Las evidencias del alcance de metas deben guardarse y organizarse cuidadosamente durante varios años (cinco años según CACECA). 
- Es recomendable fomentar una cultura de responsabilidad y excelencia en la impartición de cátedra por los docentes; tanto en docentes como en los alumnos, quienes evalúan a sus maestros.

- Organizar muy bien la información financiera por programas educativos y que ésta sea respaldada documentalmente.

- Presupuestar anualmente los ingresos y egresos por programas educativos y siempre con antelación al visto bueno del Consejo Técnico.

- Comunicación directa y constante con el departamento de planeación para agilizar la correcta atención a las recomendaciones de los organismos acreditadores.

- Se ha de generar un Plan de trabajo respecto a las metas marcadas por el organismo acreditador, haciendo partícipes a todas las personas relacionadas con el proceso.

- Se han de estudiar analíticamente las recomendaciones emitidas por el organismo acreditador, para identificar y definir responsabilidades y la fecha límite para su cumplimiento.

- Debe haber un seguimiento de actividades y apoyo constante a las personas responsables.

- Son muy positivas y motivadoras las reuniones generales de información sobre el avance de las actividades relacionadas con el proceso de acreditación.

- Se han de considerar las actividades propias de la actividad que desarrolla cada persona para no sobresaturarla de trabajo.

- Las personas asignadas a las diferentes actividades del plan de trabajo deben entregar al coordinador del departamento de planeación una carpeta bien organizada con las evidencias relacionadas con su actividad, lo que se revisa conjuntamente para subsanar errores.

- Informar y concienciar sobre la importancia de guardar evidencias.

- Integrar muy bien los expedientes relacionados con la fundamentación de cada programa educativo, el estudio de la última reestructuración, programas de estudio, perfiles de CENEVAL, y lista de instituciones que ofrecen los mismos programas educativos. 
- Mantener actualizada la información sobre las tendencias que siguen las profesiones de los programas educativos que ofrece la institución, ya que es necesaria para fundamentar los cambios en el plan de estudios.

- Apoyar el área de Informática con personal dedicado exclusivamente al diseño de los diferentes sistemas de control escolar, ya sea en calidad de prestadores de servicio social o contratados por la institución.

- Procurar la comunicación directa y constante de los responsables de informática con el departamento de Planeación y con el organismo acreditador, con el fin de evitar errores de comunicación sobre los requerimientos.

- Mejorar el proceso de transferencia de la información sobre altas y bajas de los alumnos, de la rectoría a la institución.

- La coordinación de los programas de Licenciatura se debe organizar mediante reuniones de academia quincenales.

- Todas las acciones encaminadas a solventar las recomendaciones de los organismos acreditadores han de ser respaldadas documentalmente.

- Se ha de brindar toda la información disponible sobre el proceso de acreditación a los alumnos, docentes y resto del personal.

- Estudiar detenidamente la guía que manda el organismo acreditador para conocer la metodología a seguir, saber interpretar las recomendaciones adecuadamente, y realizar de una mejor manera las autoevaluaciones previas a la visita del organismo acreditador.

- Establecer un contacto continuo con los alumnos de la institución y con todo el personal, especialmente con los encargados de los diferentes programas.

\section{Conclusiones}

La universidad pública, además de ser un espacio universal para la transmisión y renovación del conocimiento, es también una comunidad autónoma y crítica, capaz de resolver sus crisis y diferencias con acciones académicas, en un marco autocrítico para el fortalecimiento institucional.

Debido precisamente al inmovilismo, característica intrínseca de cualquier institución, es difícil para cualquiera de ellas, y en concreto la Universidad, estar preparada para enfrentar una reforma institucional. Las instituciones requieren incorporar en su vida diaria, los motivos y acciones que propicien la necesidad de un cambio. El proceso de Acreditación 
y Certificación de Carreras contribuye a establecer las bases y las necesidades propias de la innovación en todos los ámbitos de una institución educativa, puesto que propone el trabajo colegiado, la transparencia y precisión de procedimientos administrativos para el mejoramiento institucional y la construcción de una cultura de calidad, que luego disfruta toda la comunidad escolar.

La información obtenida a lo largo de nuestra investigación sobre el proceso de acreditación tiene el valor de constituir un verdadero testimonio de quienes han participado en todo el proceso, siendo así un sistema de información muy relevante para la retroalimentación de los procesos acreditadores. Los datos también permiten darse cuenta de algunas debilidades en el proceso y de las cuales están conscientes los participantes. También es importante un estudio de este tipo para externar sugerencias que pueden ser útiles a otras instituciones inmersas en procesos similares. Con base en lo anterior se concluye que:

a) Los hallazgos que se ponen de manifiesto destacan los logros obtenidos después de tres años, adoptando un modelo de gestión que privilegia el liderazgo compartido, la planeación estratégica, la autoevaluación, el trabajo en equipo, entre otros factores y cambios que impactan en una comunidad, más integrada en torno a una visión común.

b) Los avances significativos se observan en la acreditación de dos de las tres Licenciaturas, Administración y Contaduría Pública, con una planta docente con mejores niveles de profesionalización y programas de estudio, con enfoque integral, ya que los han complementado con asignaturas relacionadas con la investigación, el desarrollo de habilidades, manifestaciones culturales y el desarrollo físico a través del deporte.

c) Una organización que se compromete con un proceso de mejora continua, y la consolidación de metas a corto y largo plazo esta íntimamente relacionada con el sentido de pertenencia de sus miembros a esa organización. La corresponsabilidad para el éxito o el fracaso es también una característica en este modelo de organización.

d) Iniciar un proceso de cambio en una comunidad educativa constituye un reto que debe ser asumido por todos, sin embargo, las inercias y la experiencia considerada como conocimiento inamovible, son difíciles de vencer, para integrar una verdadera comunidad. 


\section{REFERENCIAS}

Allende, Jorge E. (2001). El impacto del avance de las Ciencias sobre la sociedad en las primeras décadas del siglo XXI. En UNESCO. Análisis de Prospectivas de la Educación en América Latina y el Caribe (pp. 27-45). Santiago de Chile: UNESCO.

Boville Luca de Tena, Belén. (2005). Seminario Diseño y Evaluación de proyectos de investigación. Programa de Doctorado en Educación: Universidad Autónoma de Tamaulipas.

Bricall, Josep M. (2000). Universidad 2 mil. Madrid: Conferencia de Rectores de las Universidades Españolas CRUE.

CACECA Consejo de acreditación en la enseñanza de la contaduría y administración A.C. (2004). Programa y Plan de Trabajo. México DF. Recuperado el 26 de Agosto de 2004 en http://www.caceca.org/archivos/html

Clark, Burton R. (1983). El sistema de educación superior. México DF: Editorial Nueva Imagen UAM - Azcapotzalco.

Didou, Sylvie. (2005). ¿Medir para conocer o para regular?: Evaluación y Acreditación de la Educación Superior en México. Avance y Perspectiva, 24(1), 31-35.

Glazman Nowalski, Raquél. (2001). Evaluación y exclusión en la enseñanza universitaria, México: Paidos Educador.

Kent, Rollin. (2002). Los temas críticos de la educación superior en América latina en los años noventa. Estudios comparativos. México: Fondo de Cultura Económica.

Lavín Santos del Prado, Jesús. (2003). Millenium III, Plan Estratégico 2003-2007. Ciudad Victoria, México: Universidad Autónoma de Tamaulipas.

Levy, Daniel C. (1995). La educación superior y el Estado en Latinoamérica. Desafíos privados al dominio público. México: Editorial Porrúa.

Mc Kernan, J. (1999). Investigación-acción y currículo. Métodos y recursos para profesionales reflexivos. Madrid: Editorial Morata.

Naishtat, Francisco, y Garcia Raggio, Ana María (2001). Filosofías de la Universidad y conflicto de racionalidades. Buenos Aires: Editorial Colihue Universidad.

Navarro, Marco Aurelio. (2000). Innovación cuesta abajo. La planeación de la educación superior 1977-1980. Ciudad Victoria, México: Universidad Autónoma de Tamaulipas.

Organización Mundial para el Comercio (OMC). (2001). Informe Anual del Director General 2001. Recuperado el 3 de febrero de 2006, de http://www.wto.org/spanish/res s/booksp s/annual report dg2001 s.pdf

Secretaría de Educación Pública (SEP) - México. (2001). Programa Nacional de Educación 2001-2006. México: SEP. Recuperado el 1 de marzo 2006, de http://www.sep.gob.mx/wb2/sep/sep 2734 programa nacional de 
Schmelkes, Corina. (1998). Manual para la Presentación de Anteproyectos e Informes de Investigación. México: Editorial Oxford.

Tyack, David y Cuban, Larry. (2000). En busca de la utopía. Un siglo de reformas de las escuelas públicas. México: FCE.

Tort Bardolet, Antoni; Sánchez-Cortés, Emilia; Carbonell Sebarroja, Jaume; Hernández, Fernando; Simó, Núria; Sancho Gil, Juana María. (1992) Para aprender de las innovaciones en los centros Revista de Educación, 299, 249-291.

Unidad Académica Multidisciplinar de Comercio y Administración Victoria (2004) Plan de Trabajo 2004. Ciudad Victoria: Universidad Autónoma de Tamaulipas.

Unidad Académica Multidisciplinar de Comercio y Administración Victoria (2005) Plan de Trabajo 2005. Ciudad Victoria: Universidad Autónoma de Tamaulipas.

Universidad Autónoma Metropolitana, Unidad Xochimilco. (1993). Planeación, Evaluación de la Universidad Pública en México. México: Colección Memorias.

Urquidi, Victor (comp.). (1996). México en la globalización. México: Fondo de Cultura Económica. 